\title{
BMJ Open End-of-life care preferences of older patients with multimorbidity: protocol of a mixed-methods systematic review
}

\author{
Ana Isabel Gonzalez-Gonzalez (1) ,1,2 Christine Schmucker, ${ }^{3}$ Julia Nothacker, ${ }^{3}$ \\ Truc Sophia Nguyen (D) ," Maria-Sophie Brueckle, ${ }^{1}$ Jeanet Blom, ${ }^{4}$ \\ Marjan van den Akker (D) , ${ }^{1}$ Kristian Röttger, ${ }^{5}$ Odette Wegwarth (D) , ${ }^{6}$ \\ Tammy Hoffmann, ${ }^{7}$ Ferdinand M Gerlach, ${ }^{8}$ Sharon E Straus, ${ }^{9}$ Joerg J Meerpohl, ${ }^{10}$ \\ Christiane Muth (1) ${ }^{8}$
}

To cite: Gonzalez-Gonzalez Al, Schmucker C, Nothacker J, et al. End-of-life care preferences of older patients with multimorbidity: protocol of a mixed-methods systematic review. BMJ Open 2020;10:e038682. doi:10.1136/ bmjopen-2020-038682

- Prepublication history and additional material for this paper are available online. To view these files, please visit the journal online (http://dx.doi. org/10.1136/bmjopen-2020038682).

Received 19 March 2020 Revised 19 May 2020

Accepted 05 June 2020

Check for updates

(C) Author(s) (or their employer(s)) 2020. Re-use permitted under CC BY-NC. No commercial re-use. See rights and permissions. Published by BMJ.

For numbered affiliations see end of article.

\section{Correspondence to} Dr Ana Isabel GonzalezGonzalez; gonzalezgonzalez@ allgemeinmedizin.uni-frankfurt. de

\section{ABSTRACT}

Introduction End-of-life care is an essential task performed by most healthcare providers and often involves decision-making about how and where patients want to receive care. To provide decision support to healthcare professionals and patients in this difficult situation, we will systematically review a knowledge cluster of the end-oflife care preferences of older patients with multimorbidity that we previously identified using an evidence map. Methods and analysis We will systematically search for studies reporting end-of-life care preferences of older patients (mean age $\geq 60$ ) with multimorbidity ( $\geq 2$ chronic conditions) in MEDLINE, CINAHL, PsycINFO, Social Sciences Citation Index, Social Sciences Citation Index Expanded, PSYNDEX and The Cochrane Library from inception to September 2019. We will include all primary studies that use quantitative, qualitative and mixed methodologies, irrespective of publication date and language.

Two independent reviewers will assess eligibility, extract data and describe evidence in terms of study/population characteristics, preference assessment method and end-of-life care elements that matter to patients (eg, lifesustaining treatments). Risk of bias/applicability of results will be independently assessed by two reviewers using the Mixed-Methods Appraisal Tool. Using a convergent integrated approach on qualitative/quantitative studies, we will synthesise information narratively and, wherever possible, quantitatively.

Ethics and dissemination Due to the nature of the proposed systematic review, ethics approval is not required. Results from our research will be disseminated at relevant (inter-)national conferences and via publication in peer-reviewed journals. Synthesising evidence on end-oflife care preferences of older patients with multimorbidity will improve shared decision-making and satisfaction in this final period of life.

PROSPERO registration number CRD42020151862.

\section{INTRODUCTION}

Multimorbidity, or the presence of multiple coexisting chronic diseases or conditions, ${ }^{1}$ affects the majority of older adults ${ }^{2}$ and is associated with increased mortality and

\section{Strengths and limitations of this study}

- This is the first systematic review on end-of-life care preferences of patients with multimorbidity and will provide an important body of evidence to support the consideration of patient-centred care in end-oflife care policy.

- A multinational and multidisciplinary team with considerable methodological experience and skills will provide the necessary expertise.

- A patient representative has been involved in designing the study to ensure that from the beginning, patient-relevant questions are assessed, and results discussed accordingly.

- The main study limitations are the poor indexing of articles and the missing or non-standardised definition of 'patient preferences'.

healthcare usage. ${ }^{3-5}$ In addition, multimorbidity negatively impacts quality of life and increases symptom burden. ${ }^{6-8}$ Evidence is therefore required on how to best manage multimorbid patients. ${ }^{9} 10$

The care of patients with multimorbidity entails complex medical decision-making especially at the end of life (EoL). EoL care refers not only to the healthcare services that are provided to patients in the final hours or days of their lives, but, more broadly, to those provided to all patients whose conditions have become advanced, progressive and incurable. ${ }^{112}$ EoL care must be embedded within the context of patient preferences, so the care that the multimorbid patients receive during the final days of their lives is concordant with the care they desire. However, individuals with multimorbidity often have to make numerous and conflicting decisions and choices, which makes eliciting their preferences rather challenging. The provision of effective EoL care to those with multimorbidity is impossible 
without cooperation between palliative care providers, speciality care and primary care. In fact, EoL care is an essential task performed by most of the healthcare providers and it often involves decision-making about how and where the patients want to receive care.

According to recent studies that were not confined to patients with multimorbidity, most adults' EoL care preferences (eg, for cardiopulmonary resuscitation) are stable over time and independent of their health status. ${ }^{13}$ A systematic review of where the adult patients would prefer to die revealed that most of the people would prefer to die at home and that such preferences are independent of changes in health status. ${ }^{14}$ These results were confirmed in another systematic review ${ }^{15}$ on adults with diverse health conditions. However, it was unclear what proportion of people preferred home when the underlying condition was taken into account (eg, cancer vs non-cancer conditions). ${ }^{15}$ Furthermore, considerable heterogeneity between and within population groups exists, both in the proportion of patients whose preferences change over time and in the direction of such changes (eg, towards or away from more aggressive care). ${ }^{10} 13$

Multimorbidity is positively associated with the desire not to be resuscitated, but this finding depends on the nature of the morbidities. Cognitive impairment, stroke and cancer were very positively associated with the desire not to be resuscitated, while heart diseases were not. ${ }^{16}$ However, we have no information on the preferences of patients with a mix of disabling/life-threatening conditions or an accumulation of several conditions. To the best of our knowledge, no systematic review has focussed on EoL care preferences of older patients with multimorbidity.

To provide decision support to healthcare providers and assist this complex patient population in an emotionally difficult situation, we aim to systematically review EoL care preferences of older patients with multimorbidity. We will base the review on a knowledge cluster of EoL care preferences that we identified in an evidence map we previously developed on health-related preferences in older patients with multimorbidity. ${ }^{17}$ The systematic review is the natural next step and will allow us to synthesise current knowledge of EoL care preferences and help prioritise and guide future innovations in EoL care policy.

\section{METHODS AND ANALYSIS}

The present protocol will follow the Preferred Reporting System Items for Systematic Review and Meta-Analysis Protocols (PRISMA-P) checklist $^{18}$ (see online supplementary additional file 1$)$.

\section{Design}

Mixed-methods systematic review using the convergent integrated approach in which data is transformed in such a way that quantitative and qualitative data can be combined and the synthesis of quantitative and qualitative studies simultaneously occurs. ${ }^{19}$

\section{Criteria for considering studies for this review}

Types of studies

We will include primary studies that use quantitative (eg, questionnaires), qualitative (eg, interviews, focus groups) and mixed-methods methodologies. Systematic reviews and meta-analyses will not be included, but if a systematic review is relevant to our topic we will screen its reference list for potentially eligible studies that were not identified in our systematic literature searches (see the section on search methods used to identify studies).

We will exclude case reports and articles, such as conference abstracts, narrative reviews and editorials.

\section{Types of participants}

We will include older patients (mean or median age $\geq 60$ years ${ }^{20}$ ) with multimorbidity (two or more simultaneous chronic conditions). ${ }^{1}$ Studies focussing on patients with one chronic disease will be included when authors have reported on at least one additional chronic condition in the majority of the study population.

Studies addressing only the preferences of caregivers, family members and healthcare professionals will be excluded. Studies confined to population-based and general public perspectives will also be excluded.

\section{Phenomenon of interest}

Our phenomenon of interest will focus on EoL care preferences, defined as preferences related to the care that should be provided in the final period of life, regardless of whether it may, in some cases, be provided for months or even years. ${ }^{12}$ EoL care preferences will comprise (i) willingness to receive life-sustaining treatments (eg, percentage of people with preferences for or against cardiopulmonary resuscitation, intubation and mechanical ventilation, intensive care, intravenous nutritional support, nasogastric tube feeding and/or dialysis withdrawal), (ii) willingness to opt for palliation of symptoms, (iii) the place where patients would prefer to receive EoL care (eg, percentage of people that would prefer to die at home) and (iv) interest in participating in a shared decision-making process related to EoL care.

We will exclude studies investigating preferences for or against interventions of limited availability or whose legal status is unclear (eg, preferences for or against euthanasia or physician-assisted suicide) as such approaches are deemed outside the scope of this review. We will also exclude studies exploring patients' will to live. (see table 1)

\section{Search methods used to identify studies Electronic searches}

We will search the following electronic sources from inception using a combination of Medical Subject Headings and keywords: MEDLINE, CINAHL, PsycINFO, Social Sciences Citation Index, Social Sciences Citation Index Expanded, PSYNDEX and The Cochrane Library. 
Table 1 Inclusion and exclusion criteria

\section{Inclusion criteria}

- Quantitative (observational and interventional) and qualitative studies addressing end-of-life care preferences from the patient's perspective

- Age: average/median age of 60 or older, geriatric patients, elderly patients

- Multimorbidity: two or more simultaneous chronic conditions

- Setting: We will not apply restrictions to geographical location, country or healthcare context

- No restrictions to the date of publication or language of the study

\section{Exclusion criteria}

- Case reports

- Articles without details of methods

- Conference abstracts

- Narrative reviews

- Editorials

- Studies investigating preferences for or against interventions that are not generally available or only legal in limited contexts (eg, euthanasia)

- Studies only addressing preferences of caregivers, family members and healthcare professionals

Population-based studies (public health perspective)
To avoid publication bias, we will not apply any restrictions to publication date or language.

We will follow the recommendations of PRESS (Peer Review of Electronic Search Strategies) and develop the final search strategy in collaboration with an expert medical science librarian. ${ }^{21}$

The electronic search strategy for the MEDLINE database from inception to September 2019 is provided in table 2. This search strategy will be adapted for use in the other databases.

\section{Searching other resources}

We will identify potentially eligible studies that are not captured by our electronic database searches by examining the reference lists of included studies, relevant systematic reviews and meta-analyses, and by carrying out

Table 2 Search for end-of-life care preferences. (13 September 2019 - MEDLINE via Ovid (medall))

1. ((advanced OR incurabl ${ }^{\star}$ OR progressive OR life-limiting OR fatal OR serious ${ }^{\star}$ OR end-stage OR terminal ${ }^{\star}$ End-of-Life adj3 (disease OR condition OR illness OR ill OR morbid*)).ti,ab,kf.

2. (End of life OR (last days adj3 life) OR (last year* adj3 life) OR (last week* adj3 life) OR (last month* adj3 life) OR (last days adj3 live) OR (last week* adj3 live) OR (last month* adj3 live) OR (last year* adj3 live) OR imminent death OR (close adj3 death) OR before death OR palliative).ti,ab,kf.

3. (Terminal Care OR Terminally III OR Hospice Care OR Life Support Care OR Advanced Cardiac Life Support OR Palliative Care).sh.

4. or/1-3

5. (Comorbidity OR Multimorbidity OR Multiple Chronic Conditions).sh.

6. ((comorbid ${ }^{*}$ OR multiple OR several OR multi OR concurrent OR complex OR more than one) adj4 (disease* OR condition* OR illness* OR morbid*)).ti,ab,kf.

7. (Comorbidit* OR multimorbidit* OR multidisease* OR polymorbid* OR frail*).ti,ab,kf.

8. $\mathrm{or} / 5-7$

9. 4 AND 8

10. (scale OR scaling OR ranking OR rating OR conjoint-analysis OR conjoint-analyses OR contingent valuation OR analytic hierarch* process* OR time trade off OR evidential reasoning OR multi-attribute utility OR maut OR multiattribute decision model OR madm OR electre iv OR electre is OR visual analog* scale OR score* OR scoring OR standard gamble OR EVIDEM OR paprika method OR simple additive weighting method OR weighted product method OR wpm OR technique for order preference by similarity to ideal solution OR topsis OR analytic network process OR anp OR todim OR macbeth OR smart OR focus group* OR interview* OR questionnair* OR choice).ti,ab,kf.

11. (prefer ${ }^{\star}$ OR wish ${ }^{\star}$ OR need OR needs OR value* OR belief* OR want ${ }^{\star}$ OR desire ${ }^{\star}$ OR priorit* OR attitude* OR Preferences perception* OR evaluation* OR choice* OR experience* OR decision* OR decide* OR perspective*).ti,ab,kf.

12. (patient* OR women* OR men ${ }^{\star}$ OR elder ${ }^{\star}$ OR old ${ }^{\star}$ OR frail*).ti,ab,kf.

13. 10 AND 11 AND 12

14. (Patient Satisfaction OR Patient Preference OR Health Priorities OR Needs Assessment OR Advance Care Planning OR Advance Directives).sh.

15. 9 AND 13

16. 9 AND 14

17. or/15-16

Methods to elicit Preferences 
searches of cited references (forward and backward citation tracking) using the Web of Science Core Collection.

\section{Study records}

\section{Data management}

Bibliographic details of all identified references will be uploaded to EndNote first and then converted into Covidence for title, abstract and full-text screening. Duplicates will be removed.

\section{Selection of studies}

Each of the two review authors (AIG-G and JN) will independently screen the title and the abstract of each identified study to determine which should be assessed further. Before screening, a stepwise calibration exercise will be performed on a sample of 30 studies, ${ }^{22}$ with the aim of achieving $80 \%$ agreement between the reviewers. In case $80 \%$ agreement is not reached, our inclusion and exclusion criteria will be refined and the calibration will be repeated until the threshold is met. We will report any changes to the inclusion and exclusion criteria that result from the calibration exercise as deviations from the published protocol. The full text of potentially eligible papers will then be retrieved and independently assessed for eligibility by the two reviewers (AIG-G and JN). Any discrepancy will be resolved through discussion and consensus by a third reviewer (CS).

We will present a PRISMA flowchart of study selection. ${ }^{23}$

\section{Data collection}

One review author (AIG-G) will extract key study and participant characteristics from all studies that fulfil the inclusion criteria and report data on the phenomenon of interest. The second review author (CS) will cross-check the extracted data. Any disagreement will be resolved by discussion, or, if necessary, by a third author (CM).

\section{Data items}

We will stratify data extraction according to the study type. Using standard extraction templates in Excel and Access datasheets, data will be extracted under the following headings: Study reference (ie, first author, year of publication, country of study origin); Study aim; Study setting; Sample size; Population characteristics (eg, age, sex, definition of multimorbidity, prognosis or illness severity, cancer or non-malignant condition); Preference-assessment method (eg, interview or questionnaire, number of assessments, time between assessments if applicable); Context of preference (ie, hypothetical/real, preference-sensitive situation); Information provided by the authors on the presentation of alternatives (eg, negative or positive framing); $;{ }^{25}$ Description of phenomenon of interest (EoL care elements that study participants were queried about, for example, resuscitation preference); and Results concerning the described phenomenon of interest (eg, proportion of participants expressing a preference for a specific type of EoL care) (table 3).
Dealing with duplicate and associated publications

In the event of multiple reports (publications) of a primary study, we will maximise the yield of information by collating all available data and using the most complete data set, aggregated across all known publications.

\section{Assessment of risk of bias in included studies}

A risk of bias ( $\mathrm{RoB})$ assessment will be conducted using the Mixed-Methods Appraisal Tool (MMAT),${ }^{26}$ whereby one author (AIG-G) will apply the MMAT criteria and a second author (CS) will verify the assessments. Both authors will discuss the impact of the RoB assessments on further analyses and involve a third author (CM) in cases of dissent. If an important RoB is detected, sensitivity analyses will be performed that exclude studies with a high RoB.

\section{Data synthesis}

We will conduct a mixed-methods systematic review using a convergent integrated approach in accordance with Joanna Briggs Institute methodology ${ }^{19}$ that will (i) synthesise qualitative data by means of thematic synthesis, (ii) synthesise quantitative data and perform meta-analysis if applicable, and in a final step, (iii) synthesise and integrate both (i) and (ii) following the methodology described by Sandelowski et al and Pearson et al. ${ }^{27}{ }^{28}$ More specifically, the approach will include the following steps:

i. Qualitative analysis and synthesis: Both reviewers (AIG-G and CS) will independently analyse the extracted data and provide thematic codes. In order to derive a matrix structure, both reviewers will discuss coding and identify overarching thematic issues and categories with the help of MaxQDA 18 software..$^{29} 30$

ii. Quantitative analysis: Data from interventional and observational studies will be analysed separately. The meta-analysis of data will be considered in studies that have provided comparable and sufficiently homogeneous outcomes. We will first assess heterogeneity qualitatively (in terms of study design, population and the phenomenon of interest). Assuming the qualitative assessment does not preclude metaanalyses of studies, we will also assess heterogeneity by means of $\mathrm{X}^{2}$ and additional tests. If a meta-analysis is impossible, a descriptive analysis will be carried out.

iii. Mixed-methods data synthesis (integrated synthesis methodology ${ }^{2728}$ ): To synthesise qualitative and quantitative data, three reviewers (AIG-G, CS and CM) will decide which is the most promising compatible format based on the results of (i) and (ii), whereby the decision will depend mainly on the number of qualitative and quantitative studies that are eligible for inclusion. Afterwards, ${ }^{27}$ data will either be classified according to subject matter (resulting in data synthesis by means of meta-aggregation) or by converting qualitative data into a numerical format (resulting in a quantitative synthesis using meta-analytical approaches). 27283132 
Table 3 Data extraction framework

\begin{tabular}{lll}
\hline Bibliometrics & Description & Coding \\
\hline Study identification & First author, year of publication & (journal's description) \\
Study characteristics & Study aim & (authors' description) \\
& Geographical location & Country \\
& Study setting & Inpatient, outpatient \\
& Type of study & Observational (ie, qualitative, quantitative cross- \\
& & sectional, quantitative longitudinal, mixed methods) \\
& & or interventional study
\end{tabular}

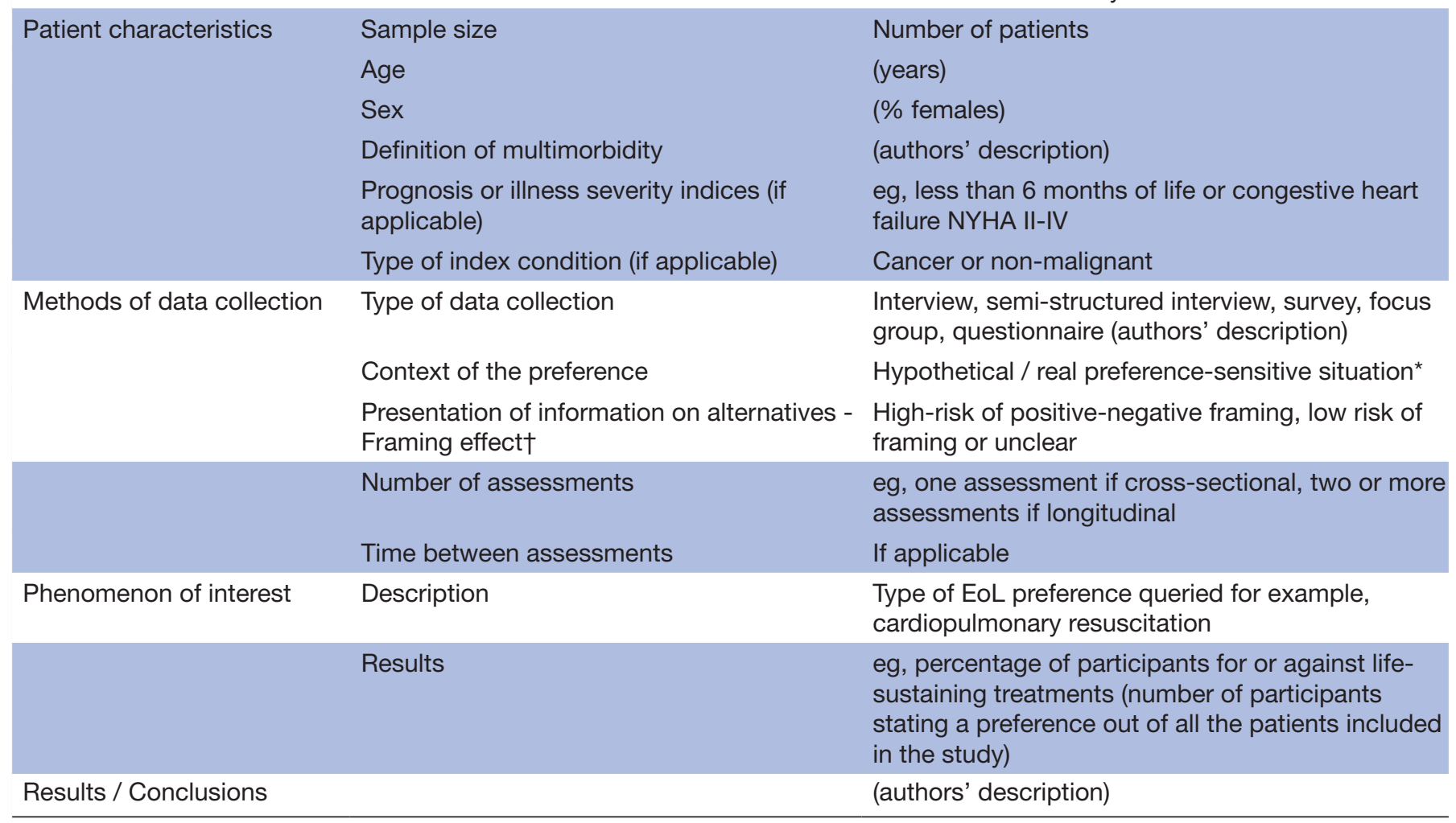

*Hypothetical preference-sensitive situation: EoL care preferences are measured by asking study participants to imagine themselves in a situation in the future that requires such care; Real preference-sensitive situation: EoL care preferences are measured by asking study participants to state their preferences in a context that actually requires them to express a preference for such care. Examining preferences using hypothetical scenarios removes the acute stress of making decisions when confronted with an EoL situation.

†Framing effect: Cognitive bias caused by the influence of the way information is presented on the choices people make.

EoL, End of Life; NYHA, New York Heart Association.

\section{Planned sensitivity and subgroup analysis}

If the available data allows, we will conduct sensitivity analyses that exclude studies at high risk of bias in order to determine its impact. In addition, we plan to conduct subgroup analyses to examine whether EoL care preferences are affected by sex, specific preference assessment contexts (hypothetical or real scenarios), the type of advanced disease (cancer or non-malignant) and patient prognosis or illness severity. If the included studies do not permit quantitative synthesis, we will descriptively report on evidence relating to the above-mentioned aspects.

\section{Timeline for review}

At the time of this submission we have already completed electronic searches, piloted the study selection process and started formally screening search results with respect to the eligibility criteria. This systematic review is scheduled to end in August 2020.

\section{Patient and public involvement}

A patient representative (KR) from the Federal Joint Committee 'Gemeinsamer Bundesausschuss (G-BA)' actively participated in the design of the systematic review. He was involved in defining the research question, selecting the methodology to be used and the data to be collected, as well as selecting the phenomenon of interest. $\mathrm{KR}$ will also be involved in the analysis and interpretation of the findings, crafting the overall message, the development of recommendations and in the dissemination of the results. KR has considerable experience in evidencebased medicine and an understanding of the pivotal role of patients' preferences in the provision of healthcare. 
The G-BA is the ultimate decision-making body for the joint self-administration of stakeholders in the German health service and the statutory health insurance service catalogue for over 70 million insured individuals is based on its guidelines.

\section{ETHICS AND DISSEMINATION}

Due to the nature of the proposed systematic review, ethics approval is not required. We will disseminate our study findings to healthcare providers and patients and present them at relevant national and international conferences. We also aim to publish the results of the study in a peerreviewed journal.

\section{Author affiliations}

${ }^{1}$ Institute of General Practice, Goethe University, Frankfurt am Main, Germany

${ }^{2}$ Red de Investigación en Servicios de Salud en Enfermedades Crónicas (REDISSEC), Madrid, Spain

${ }^{3}$ Institute for Evidence in Medicine (for Cochrane Germany Foundation), Medical Center, Faculty of Medicine, University of Freiburg, Freiburg, Germany

${ }^{4}$ Department of Public Health and Primary Care, Leiden University Medical Center, Leiden, Netherlands

${ }^{5}$ Federal Joint Committee "Gemeinsamer Bundesausschuss", Berlin, Germany ${ }^{6}$ Center for Adaptive Rationality, Max Planck Institute for Human Development, Berlin, Germany

${ }^{7}$ Institute for Evidence-Based Healthcare, Faculty of Health Sciences and Medicine, Bond University, Gold Coast, Queensland, Australia

${ }^{8}$ Institute of General Practice, Goethe University, Frankfurt, Germany

${ }^{9}$ Department of Medicine, University of Toronto, Toronto, Ontario, Canada

${ }^{10}$ Institute for Evidence in Medicine (for Cochrane Germany Foundation), Medical Center-University of Freiburg, Freiburg, Germany

Acknowledgements The authors would like to thank Kiran Chapidi for his support as data manager. We would also like to thank Phillip Elliott for editing the manuscript.

Contributors AIG-G wrote the initial draft of the protocol. CM is the guarantor of the review. CS and JJM provided methodological guidance and revisions of the manuscript. CS and $\mathrm{JN}$ assisted in the identification of databases and reviewed the search strategy. TSN, M-SB, JB, MvdA, KR, OW, TH, SES and FMG are cosupervisors of this project, provided advice at all stages of the development of the protocol and contributed to the revision of the manuscript. All authors read and approved the final manuscript.

Funding This work was supported by the German Federal Ministry of Education and Research, grant number 01GL1729. The funder had no role in developing the protocol for this review.

Competing interests None declared.

Patient and public involvement Patients and/or the public were involved in the design, or conduct, or reporting, or dissemination plans of this research. Refer to the Methods section for further details.

Patient consent for publication Not required.

Provenance and peer review Not commissioned; externally peer reviewed.

Open access This is an open access article distributed in accordance with the Creative Commons Attribution Non Commercial (CC BY-NC 4.0) license, which permits others to distribute, remix, adapt, build upon this work non-commercially, and license their derivative works on different terms, provided the original work is properly cited, appropriate credit is given, any changes made indicated, and the use is non-commercial. See: http://creativecommons.org/licenses/by-nc/4.0/.

\section{ORCID iDs}

Ana Isabel Gonzalez-Gonzalez http://orcid.org/0000-0002-1707-0596

Truc Sophia Nguyen http://orcid.org/0000-0002-9774-6751

Marjan van den Akker http://orcid.org/0000-0002-1022-8637

Odette Wegwarth http://orcid.org/0000-0003-0885-2673

Christiane Muth http://orcid.org/0000-0001-8987-182X

\section{REFERENCES}

1 van den Akker M, Buntinx F, Knottnerus JA. Comorbidity or multimorbidity. Eur J Gen Pract 1996;2:65-70.

2 Fortin M, Bravo G, Hudon C, et al. Prevalence of multimorbidity among adults seen in family practice. Ann Fam Med 2005;3:223-8.

3 Marengoni A, Angleman S, Melis R, et al. Aging with multimorbidity: a systematic review of the literature. Ageing Res Rev 2011;10:430-9.

4 Wallace E, Hinchey T, Dimitrov BD, et al. A systematic review of the probability of repeated admission score in community-dwelling adults. J Am Geriatr Soc 2013;61:357-64.

5 Glynn LG, Valderas JM, Healy P, et al. The prevalence of multimorbidity in primary care and its effect on health care utilization and cost. Fam Pract 2011;28:516-23.

6 Fortin M, Lapointe L, Hudon C, et al. Multimorbidity and quality of life in primary care: a systematic review. Health Qual Life Outcomes 2004;2:51.

7 Fortin M, Bravo G, Hudon C, et al. Relationship between multimorbidity and health-related quality of life of patients in primary care. Qual Life Res 2006;15:83-91.

8 Rosbach M, Andersen JS. Patient-experienced burden of treatment in patients with multimorbidity - A systematic review of qualitative data. PLoS One 2017;12:e0179916.

9 Muth C, Blom JW, Smith SM, et al. Evidence supporting the best clinical management of patients with multimorbidity and polypharmacy: a systematic guideline review and expert consensus. J Intern Med 2019;285:272-88.

10 Campbell-Scherer D. Multimorbidity: a challenge for evidence-based medicine. Evid Based Med 2010;15:165-6.

11 National Institute for Health and Care Excellence (NICE). Care of dying adults in the last days of life: NICE guideline, 2015. Available: https://www.nice.org.uk/guidance/ng31/resources/care-of-dyingadults-in-the-last-days-of-life-pdf-1837387324357 [Accessed 27 Feb 2020].

12 National Institute for Health and Care Excellence (NICE). End of life care for adults: service delivery: NICE guideline, 2019. Available: https://www.nice.org.uk/guidance/ng142/resources/end-of-life-carefor-adults-service-delivery-pdf-66141776457925 [Accessed 27 Feb 2020].

13 Auriemma CL, Nguyen CA, Bronheim R, et al. Stability of end-of-life preferences: a systematic review of the evidence. JAMA Intern Med 2014;174:1085-92.

14 Gomes B, Calanzani N, Gysels M, et al. Heterogeneity and changes in preferences for dying at home: a systematic review. BMC Palliat Care 2013;12:7.

15 Hoare S, Morris ZS, Kelly MP, et al. Do patients want to die at home? A systematic review of the UK literature, focused on missing preferences for place of death. PLoS One 2015;10:e0142723.

16 de Decker L, Annweiler C, Launay C, et al. Do not resuscitate orders and aging: impact of multimorbidity on the decision-making process. $J$ Nutr Health Aging 2014;18:330-5.

17 Gonzalez Al, Schmucker C, Nothacker J, et al. Health-related preferences of older patients with multimorbidity: an evidence MAP. BMJ Open 2019;9:e034485.

18 Shamseer L, Moher D, Clarke M, et al. Preferred reporting items for systematic review and meta-analysis protocols (PRISMA-P) 2015: elaboration and explanation. BMJ 2015;350:g7647.

19 Lizarondo L, Stern C, Carrier J, et al. Mixed methods systematic reviews. In: Aromataris E, Munn Z, eds. Joanna Briggs Institute reviewer's manual, 2017. https://reviewersmanual.joannabriggs. org/

20 WHO. Ageing and health, 2018. Available: https://www.who.int/ news-room/fact-sheets/detail/ageing-and-health [Accessed 14 May 2020].

21 McGowan J, Sampson M, Salzwedel DM, et al. PRESS Peer Review of Electronic Search Strategies: 2015 Guideline Explanation and Elaboration (PRESS E\&E). Ottawa, 2016.

22 Browne $\mathrm{RH}$. On the use of a pilot sample for sample size determination. Stat Med 1995;14:1933-40.

23 Moher D, Liberati A, Tetzlaff J, et al. Preferred reporting items for systematic reviews and meta-analyses: the PRISMA statement. PLoS Med 2009;6:e1000097.

24 Vélez Ortiz D, Martinez RO, Espino DV. Framing effects on endof-life preferences among Latino elders. Soc Work Health Care 2015;54:708-24.

25 Tversky A, Kahneman D. The framing of decisions and the psychology of choice. Science 1981;211:453-8.

26 Pluye P, Hong QN. Combining the power of stories and the power of numbers: mixed methods research and mixed studies reviews. Annu Rev Public Health 2014;35:29-45.

27 Sandelowski M, Voils Cl, Barroso J. Defining and designing mixed research synthesis studies. Res Sch 2006;13:29. 
28 Pearson A, White H, Bath-Hextall F, et al. A mixed-methods approach to systematic reviews. Int J Evid Based Healthc 2015;13:121-31

29 Thomas J, Harden A. Methods for the thematic synthesis of qualitative research in systematic reviews. BMC Med Res Methodol 2008;8:45.

30 Booth A, Noyes J, Flemming K, et al. Structured methodology review identified seven (retreat) criteria for selecting qualitative evidence synthesis approaches. J Clin Epidemiol 2018;99:41-52.
31 Harden A, Thomas J, Cargo M, et al. Cochrane qualitative and implementation methods group guidance series-paper 5 : methods for integrating qualitative and implementation evidence within intervention effectiveness reviews. J Clin Epidemiol 2018;97:70-8

32 Noyes J, Booth A, Moore G, et al. Synthesising quantitative and qualitative evidence to inform guidelines on complex interventions: Clarifying the purposes, designs and outlining some methods. BMJ Glob Health 2019;4:e000893. 